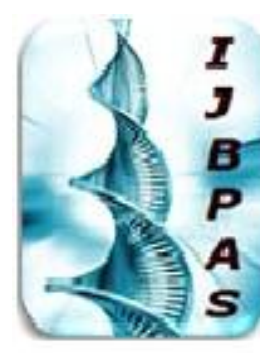

International Journal of Biology, Pharmacy

and Allied Sciences (IJBPAS)

'A Bridge Betusen Caboratory and Qundo'

Www.iibpas.com

\title{
SELF-SUFFICIENCY MODEL OF TILAPIA AQUACULTURE INMINALIN, PAMPANGA, PHILIPPINES
}

\author{
ALVIN T. REYES* \\ College of Fisheries-Freshwater Aquaculture Center, Central Luzon State University, Science City of \\ Muñoz, Nueva Ecija, Philippines \\ *Corresponding author: Alvin T. Reves; E-mail: alvinreves1845@gmail.com \\ Received 13 ${ }^{\text {th }}$ Oct. 2018; Revised $13^{\text {th }}$ Nov. 2018; Accepted $24^{\text {th }}$ Nov. 2018; Available online $1^{\text {st }}$ March 2019 \\ https://doi.org/10.31032/IJBPAS/2019/8.3.4666
}

\begin{abstract}
This study was conducted in order to create a self-sufficiency model of tilapia aquaculture in Minalin, Pampanga, Philippines. The specific objectives of this study include the following: (1) To dynamically visualize the effects of compounding factors on tilapia production and consumption in the municipality; (2) To forecast local tilapia sufficiency over a given period of time; and (3) To identify interventions that would improve tilapia self-sufficiency in the municipality.

The study used secondary data from published researches and from official websites of government offices in the Philippines. The researcher utilized STELLA Version 9.02in order to dynamically visualize and communicate the self-sufficiency model of tilapia aquaculture in the municipality.

Available tilapia for local consumption was negatively affected by increasing population, change in capita consumption towards fish protein, reduction of available land for tilapia farming, poor water quality, diseases and extreme weather events. In order to lessen the compounding effects of these factors, interventions such as technology adoption, management of population growth and reduction of land conversion rate were deemed effective. The local tilapia sufficiency was prolonged from 16 years to 19 years because of these interventions. In addition, 50\% reduction in land conversion rate could extend the local sufficiency of tilapia until the $26^{\text {th }}$ year.
\end{abstract}

Keywords: Self-sufficiency model, tilapia, STELLA, aquaculture 


\section{INTRODUCTION}

Aquaculture is now a growing business around the world contributing to a large percentage of the total world fish production. In 2011, its world production of 62.7 million tons has increased by $6.2 \%$ from 2010 [1]. In the Philippines, the province of Pampanga has become a major centre of production with its polyculture system, associating the shrimp with milkfish and sometimes tilapia and/or crabs [2]. Forty percent of tilapia production in Central Luzon has been supplied by Pampanga [3] .

According to the World Health Organization (2003) [4], around 13-16\% of total animal protein consumed by human is aquatic in origin. Tilapia in the Philippines is one of the main sources of protein for its continuously growing population because of its easy digestibility and high nutritional value [5].

Minalin is a fourth class highly urbanized municipality in the province of Pampanga and is located southwest of the capital city of San Fernando. It consists of 15 barangays with a total land area of 4,827 ha. Almost $57 \%$ of its land area is used for aquaculture activities. Minalin is known as producer of tilapia, milkfish, shrimp and crab [6]. As of 2014, Minalin has 1,755 pond units used for tilapia aquaculture [7].
Tilapia aquaculture in Minalin, Pampanga is likely to become even more important as populations continue to increase, as the quality of environment degrades, and the pressures on scarce land for agriculture continue to grow, pushing more people towards aquaculture as a 'lastresort' activity. In addition, tilapia production is affected by multitude of factors and their inherent interactions [7].

The "Self-sufficiency Model of Tilapia Aquaculture in Minalin, Pampanga, Philippines" has tried to answer these two central questions: (1) What are the compounding effects of increasing rate of land conversion and population growth, increasing preference for fish-protein source, poor water quality, adverse weather conditions, diseases, and adoption of new technologies and other interventions on local tilapia sufficiency?; and (2) Given the multitude of factors that affect tilapia production and consumption, does the municipality maintains its self-sufficiency over a period of time?

The general objective of this paper is to create a self-sufficiency model of tilapia aquaculture in Minalin, Pampanga, Philippines. Specific objectives include: (1) To dynamically visualize the effects of 
compounding factors on tilapia production and consumption in the municipality; (2) To forecast local tilapia sufficiency over a given period of time; and (3) To identify interventions that would improve tilapia selfsufficiency in the municipality.

\section{MATERIALS AND METHOD}

The study used secondary data from the project "Development of Comprehensive Geo-referenced Database for Ecological Risk Analyses of Tilapia Pond Culture in Pampanga" of the Freshwater Aquaculture
Center in Central Luzon State University (FAC-CLSU). Other information needed in this study was searched in the official websites of government offices in the Philippines and from published researches. In order to dynamically visualize and communicate the "Self-sufficiency Model of Tilapia Aquaculture in Minalin, Pampanga, Philippines", the researcher used STELLA Version 9.02.The DPSIR (Driver - Pressure - State - Impact - Response) framework is provided in Figure 1.

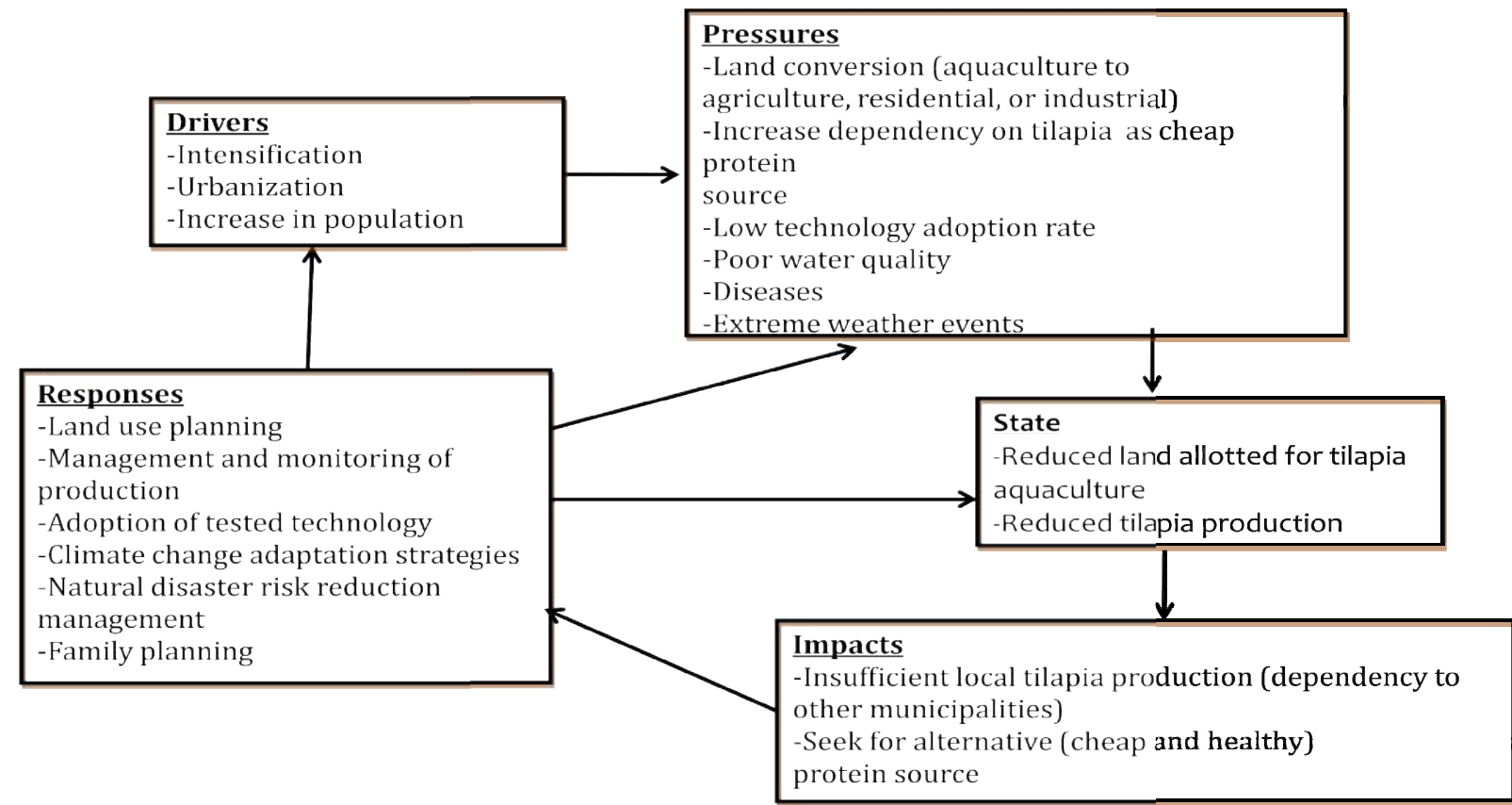

Figure 1: DPSIR framework for the sufficiency model of tilapia aquaculture in Minalin, Pampanga, Philippines

\section{RESULTS AND DISCUSSION}

Provided in Table 1 are the variables that were considered in the creation of conceptual models. Majority of the variables used were based upon actual datagenerated 
from government offices and research centers.

Shown in Figure 2 is the selfsufficiency model of tilapia aqauculture in Minalin, Pampanga,Philippines using STELLA Version 9.02. Variables affecting local tilapia production are land area allotted for production (1,293.08 ha), production rate $(10,740 \mathrm{~kg} / \mathrm{ha} /$ year $)$ and extreme weather event (reduction by $3.26 \%$ ). Meanwhile, tilapia consumption is affected by population (44,001), per capita consumption (31 $\mathrm{kg} /$ year) and change in per capita consumption (4\%). In this model, interventions (Table 1) that could positively affect tilapia sufficiency in the locality were not included.

Reduction in land alloted for tilapia faming coupled with increasing population, disease outbreaks and extreme weather condition (typhoon) could lead to reduction of available tilapia for local consumption in the long run (Figure 2; Table 2). For the next 16 years, tilapia produced is always higher than tilapia consumed and this scenario would result to accumulation of surplus production (blue line in Figure 3; Table 2). If the author assume that tilapia is not a perishable good and surplus production is kept in the municipality, it appeared that local tilapia sufficiency is to be experienced until the next 44 years (Figure 3; Table 2). In reality, tilapia is considered a perishable good and surplus production will be marketed outside the municipality. Using the latter scenario, starting at its $17^{\text {th }}$ year, tilapia consumed is higher than tilapia produced. Hence, Minalin will import tilapia from the nearby municipalities of Pampanga starting at 239.70 tons (Figure 4; Table 2).

In Figure 5, interventions to improve production rate (e.g. technology adoption), to reduce land conversion rate (e.g. evaluation of Comprehensive Land Use Plan or CLUP, financial support from government) and to manage population growth rate (e.g. family planning, delimit migration in the municipality) were incorporated.

Based on studies conducted by FACCLSU, the use of sex-reversed tilapia and probiotics could increase the production by approximately $10 \%$. Family planning program of the municipality for instance was effective in reducing population growth rate by $10 \%$. Lastly, land conversion rate was reduced by $25 \%$ because of effort and assistance extended by the local and provincial government (Figure 6). Using the following assumptions, it turned out that local supply of tilapia for consumption was sufficient until the next 19 years. Minalin 
will import tilapia starting at 75.84 tons

(Figures 7 and 8; Table 3).

The author also tried to reduce the land conversion rate up to $50 \%$ and the other two interventions were remained constant
(Figure 8). Result showed that local tilapia sufficiency was prolonged to 26 years. For the next year, Minalin will import tilapia starting at 191.35 tons (Figures 9 and 10; Table 4).

Table 1: Variables used in the creation of self-sufficiency model of tilapia aquaculture in Minalin, Pampanga, Philippines

\begin{tabular}{|c|c|c|}
\hline Variables & Value & Source \\
\hline Average productive area for tilapia farming (2012-2014) & $1,293.08$ ha & FAC-CLSU \\
\hline $\begin{array}{l}\text { Average tilapia production } \\
(2012-2014)\end{array}$ & $10,740 \mathrm{~kg} / \mathrm{ha} /$ year & FAC-CLSU \\
\hline $\begin{array}{l}\text { Reduction of production due to: } \\
\text { Land conversion (2000-2014) } \\
\text { Poor water quality } \\
\quad \text { Diseases } \\
\text { Extreme weather events (Typhoon Yolanda on Central } \\
\text { Luzon, 2013) }\end{array}$ & $\begin{array}{c}10-12 \% \\
12-15 \% \\
10 \% \\
3.26 \%\end{array}$ & $\begin{array}{c}\text { Published report } \\
\text { Published report } \\
\text { World Organization for } \\
\text { Animal Health } \\
\text { Bureau of Agricultural } \\
\text { Statistics (BAS) }\end{array}$ \\
\hline $\begin{array}{l}\text { Increase of production due to: } \\
\text { Technology adoption } \\
\text { Reduction on land conversion rate }\end{array}$ & $\begin{array}{c}5-10 \% \\
25 \%, 50 \%\end{array}$ & $\begin{array}{l}\text { FAC-CLSU } \\
\text { Assumption }\end{array}$ \\
\hline Population (2010) & 44,001 people & $\begin{array}{l}\text { Philippine Statistics } \\
\text { Authority (PSA) }\end{array}$ \\
\hline Birth rate (2007-2010) & $3.45 \%$ & PSA \\
\hline $\begin{array}{l}\text { Reduction of consumption due to: } \\
\text { Reduction onpopulation growth rate }\end{array}$ & $10 \%$ & Assumption \\
\hline Per capita consumption of tilapia in Pampanga (2012) & $31 \mathrm{~kg} / \mathrm{yr}$ & Assumption \\
\hline Change in fish capita consumption (2003-2011) & $4 \%$ & $\begin{array}{c}\text { Bureau of Fisheries and } \\
\text { Aquatic Resources (BFAR) }\end{array}$ \\
\hline
\end{tabular}

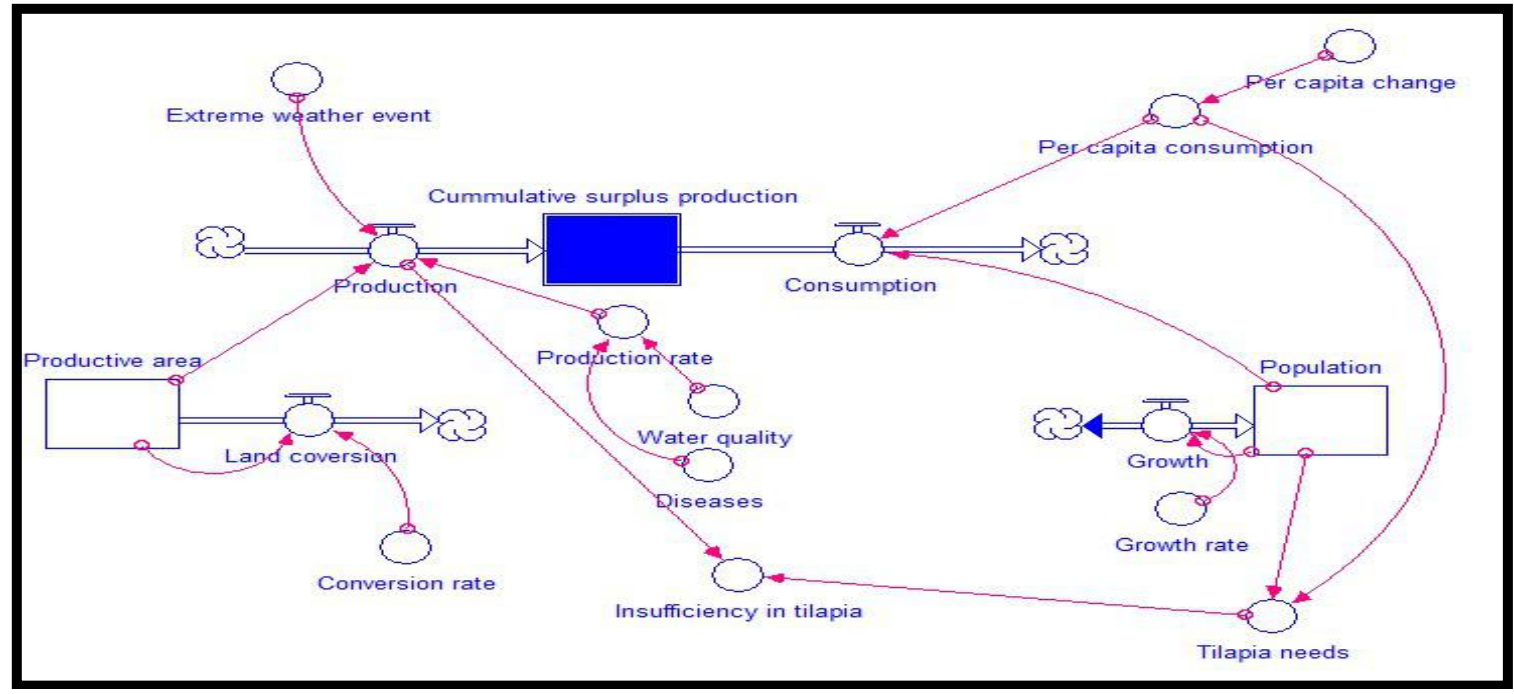

Figure 2: Local tilapia sufficiency model without interventions 


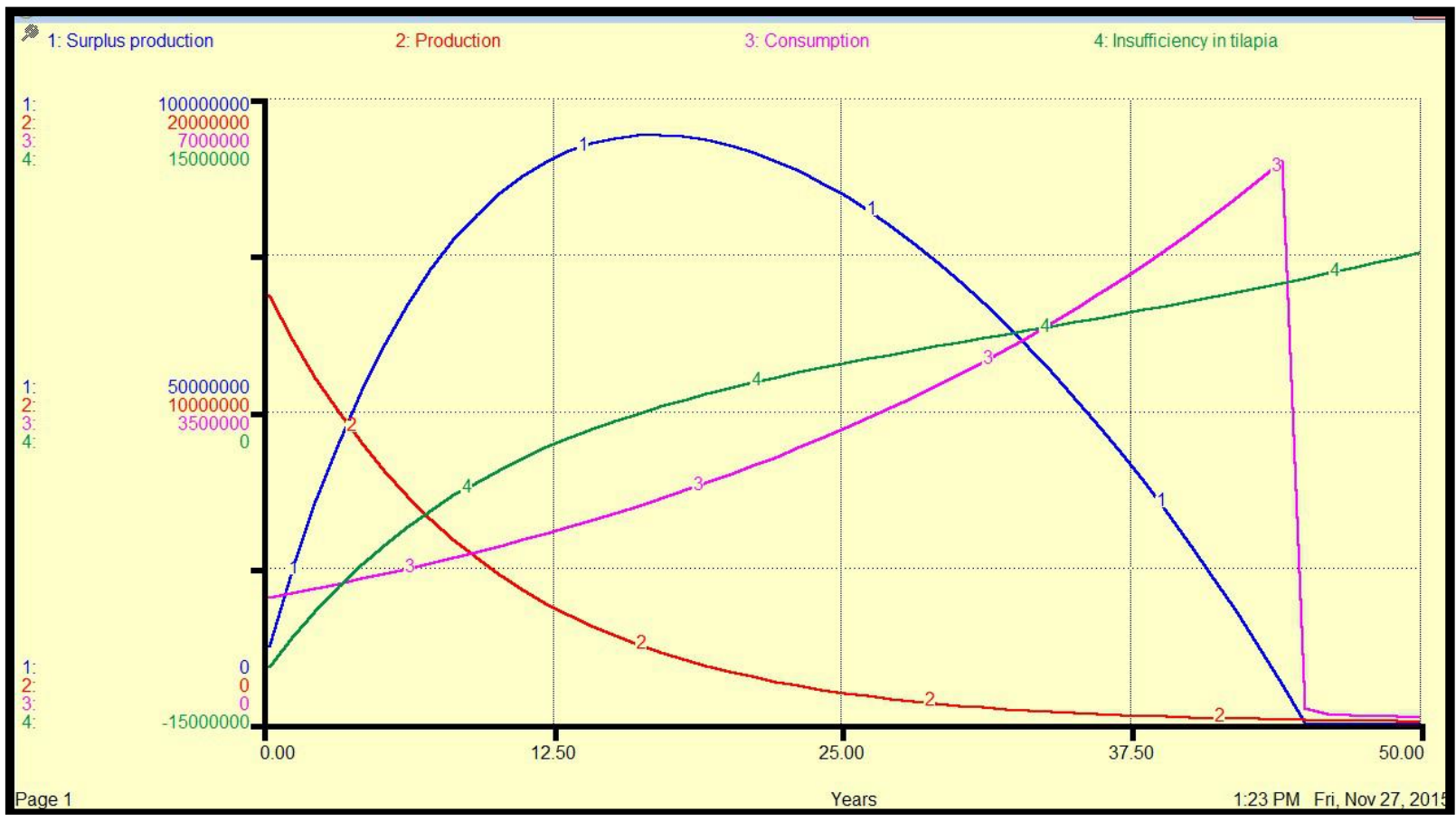

Figure 3: Cumulative surplus production and insufficiency in available tilapia over time

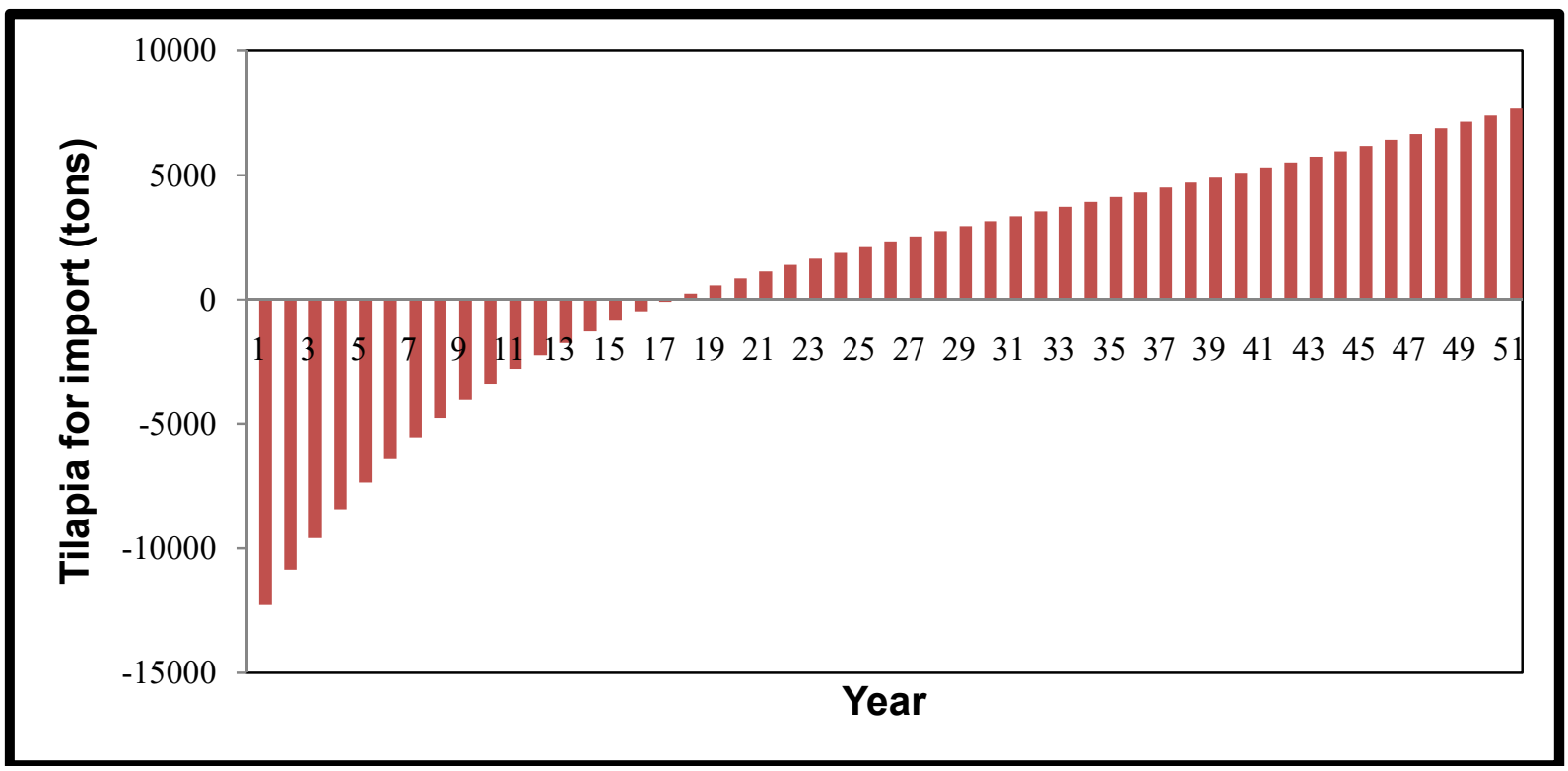

Figure 4: The municipality will start to import tilapia on the $17^{\text {th }}$ year 
Table 2: Tabular result of the model without interventions

\begin{tabular}{|c|c|c|c|c|c|}
\hline Year & $\begin{array}{l}\text { Productive } \\
\text { Area (ha) }\end{array}$ & $\begin{array}{c}\text { Production } \\
(\mathrm{kg})\end{array}$ & $\begin{array}{c}\text { Consumption } \\
(\mathrm{kg})\end{array}$ & $\begin{array}{l}\text { Cumulative Surplus } \\
\text { Production (kg) }\end{array}$ & Insufficiency (kg) \\
\hline $\mathbf{0}$ & $1,293.08$ & $13,703,639.68$ & $1,418,592.24$ & $12,285,047.44$ & $-12,285,047.44$ \\
\hline 1 & $1,163.77$ & $12,333,275.71$ & $1,467,533.67$ & $24,570,094.87$ & $-10,865,742.04$ \\
\hline 2 & $1,047.39$ & 11,099,948.14 & $1,518,163.58$ & $35,435,836.91$ & $\mathbf{- 9 , 5 8 1 , 7 8 4 . 5 5}$ \\
\hline 3 & 942.66 & $\mathbf{9 , 9 8 9 , 9 5 3 . 3 2}$ & $1,570,540.23$ & $45,017,621.46$ & $-8,419,413.10$ \\
\hline 4 & 848.39 & $8,990,957.99$ & $1,624,723.87$ & $53,437,034.55$ & $-7,366,234.13$ \\
\hline 5 & 763.55 & $8,091,862.19$ & $1,680,776.84$ & $60,803,268.68$ & $-6,411,085.35$ \\
\hline 6 & 687.2 & $7,282,675.97$ & $1,738,763.64$ & $67,214,354.03$ & $-5,543,912.33$ \\
\hline 7 & 618.48 & $6,554,408.38$ & $1,798,750.99$ & $72,758,266.37$ & $-4,755,657.39$ \\
\hline 8 & 556.63 & $5,898,967.54$ & $1,860,807.89$ & $77,513,923.76$ & $-4,038,159.64$ \\
\hline 9 & 500.97 & $5,309,070.78$ & $\mathbf{1 , 9 2 5 , 0 0 5 . 7 7}$ & $81,552,083.40$ & $-3,384,065.02$ \\
\hline 10 & 450.87 & $4,778,163.71$ & $1,991,418.47$ & $84,936,148.42$ & $-2,786,745.24$ \\
\hline 11 & 405.78 & $4,300,347.34$ & $2,060,122.40$ & 87,722,893.66 & $-2,240,224.93$ \\
\hline 12 & 365.2 & $3,870,312.60$ & $2,131,196.63$ & $89,963,118.59$ & $-1,739,115.98$ \\
\hline 13 & 328.68 & $3,483,281.34$ & $2,204,722.91$ & $91,702,234.57$ & $-1,278,558.43$ \\
\hline 14 & 295.82 & $3,134,953.21$ & $2,280,785.85$ & $92,980,793.00$ & $-854,167.36$ \\
\hline 15 & 266.23 & $2,821,457.89$ & $2,359,472.96$ & $93,834,960.36$ & $-461,984.93$ \\
\hline 16 & 239.61 & $2,539,312.10$ & $2,440,874.78$ & $94,296,945.28$ & $-98,437.32$ \\
\hline 17 & 215.65 & $2,285,380.89$ & $2,525,084.96$ & $94,395,382.60$ & $239,704.07$ \\
\hline 18 & 194.08 & $2,056,842.80$ & $2,612,200.39$ & $94,155,678.53$ & $555,357.59$ \\
\hline 19 & 174.68 & $1,851,158.52$ & $2,702,321.30$ & $93,600,320.94$ & $851,162.78$ \\
\hline 20 & 157.21 & $1,666,042.67$ & $2,795,551.39$ & $92,749,158.16$ & $1,129,508.72$ \\
\hline 21 & 141.49 & $1,499,438.40$ & $2,891,997.91$ & $91,619,649.44$ & $1,392,559.51$ \\
\hline 22 & 127.34 & $1,349,494.56$ & $2,991,771.84$ & $90,227,089.93$ & $1,642,277.28$ \\
\hline 23 & 114.6 & $1,214,545.10$ & $3,094,987.97$ & $88,584,812.65$ & $1,880,442.86$ \\
\hline 24 & 103.14 & $1,093,090.59$ & $3,201,765.05$ & $86,704,369.78$ & $2,108,674.46$ \\
\hline 25 & 92.83 & $983,781.53$ & $3,312,225.95$ & $84,595,695.33$ & $2,328,444.41$ \\
\hline 26 & 83.55 & $885,403.38$ & $3,426,497.74$ & $82,267,250.92$ & $2,541,094.36$ \\
\hline 27 & 75.19 & $796,863.04$ & $3,544,711.91$ & $79,726,156.56$ & $2,747,848.87$ \\
\hline 28 & 67.67 & $717,176.74$ & $3,667,004.47$ & $76,978,307.68$ & $2,949,827.74$ \\
\hline 29 & 60.91 & $645,459.06$ & $3,793,516.13$ & $74,028,479.95$ & $3,148,057.06$ \\
\hline 30 & 54.82 & $580,913.16$ & $3,924,392.44$ & $70,880,422.89$ & $3,343,479.28$ \\
\hline 31 & 49.33 & $522,821.84$ & $4,059,783.97$ & $67,536,943.61$ & $3,536,962.13$ \\
\hline 32 & 44.4 & $470,539.66$ & $4,199,846.52$ & $63,999,981.48$ & $3,729,306.86$ \\
\hline 33 & 39.96 & $423,485.69$ & $4,344,741.23$ & $60,270,674.61$ & $3,921,255.53$ \\
\hline 34 & 35.96 & $381,137.12$ & $4,494,634.80$ & $56,349,419.08$ & $4,113,497.68$ \\
\hline 35 & 32.37 & $343,023.41$ & $4,649,699.70$ & $52,235,921.40$ & $4,306,676.29$ \\
\hline 36 & 29.13 & $308,721.07$ & $4,810,114.34$ & $47,929,245.12$ & $4,501,393.27$ \\
\hline 37 & 26.22 & $277,848.96$ & $4,976,063.28$ & 43,427,851.85 & $4,698,214.32$ \\
\hline 38 & 23.6 & $250,064.07$ & $5,147,737.47$ & $38,729,637.53$ & $4,897,673.40$ \\
\hline 39 & 21.24 & $225,057.66$ & $5,325,334.41$ & $33,831,964.13$ & $5,100,276.75$ \\
\hline 40 & 19.11 & $202,551.89$ & $5,509,058.45$ & $28,731,687.38$ & $5,306,506.55$ \\
\hline 41 & 17.2 & $182,296.70$ & $5,699,120.96$ & $23,425,180.82$ & $5,516,824.26$ \\
\hline 42 & 15.48 & $164,067.03$ & $5,895,740.64$ & $17,908,356.57$ & $5,731,673.60$ \\
\hline 43 & 13.93 & $147,660.33$ & $6,099,143.69$ & $12,176,682.96$ & $5,951,483.36$ \\
\hline 44 & 12.54 & $132,894.30$ & $6,309,564.15$ & $6,225,199.61$ & $6,176,669.85$ \\
\hline 45 & 11.29 & $119,604.87$ & $168,134.63$ & $48,529.76$ & $6,407,639.24$ \\
\hline 46 & 10.16 & $107,644.38$ & $107,644.38$ & $\mathbf{0}$ & $6,644,789.65$ \\
\hline 47 & 9.14 & $96,879.94$ & $96,879.94$ & $\mathbf{0}$ & 6,888,513.06 \\
\hline 48 & 8.23 & $87,191.95$ & $87,191.95$ & $\mathbf{0}$ & $7,139,197.11$ \\
\hline 49 & 7.4 & $78,472.75$ & $78,472.75$ & $\mathbf{0}$ & $7,397,226.73$ \\
\hline Final & 6.66 & & & $\mathbf{0}$ & $7,662,985.64$ \\
\hline
\end{tabular}




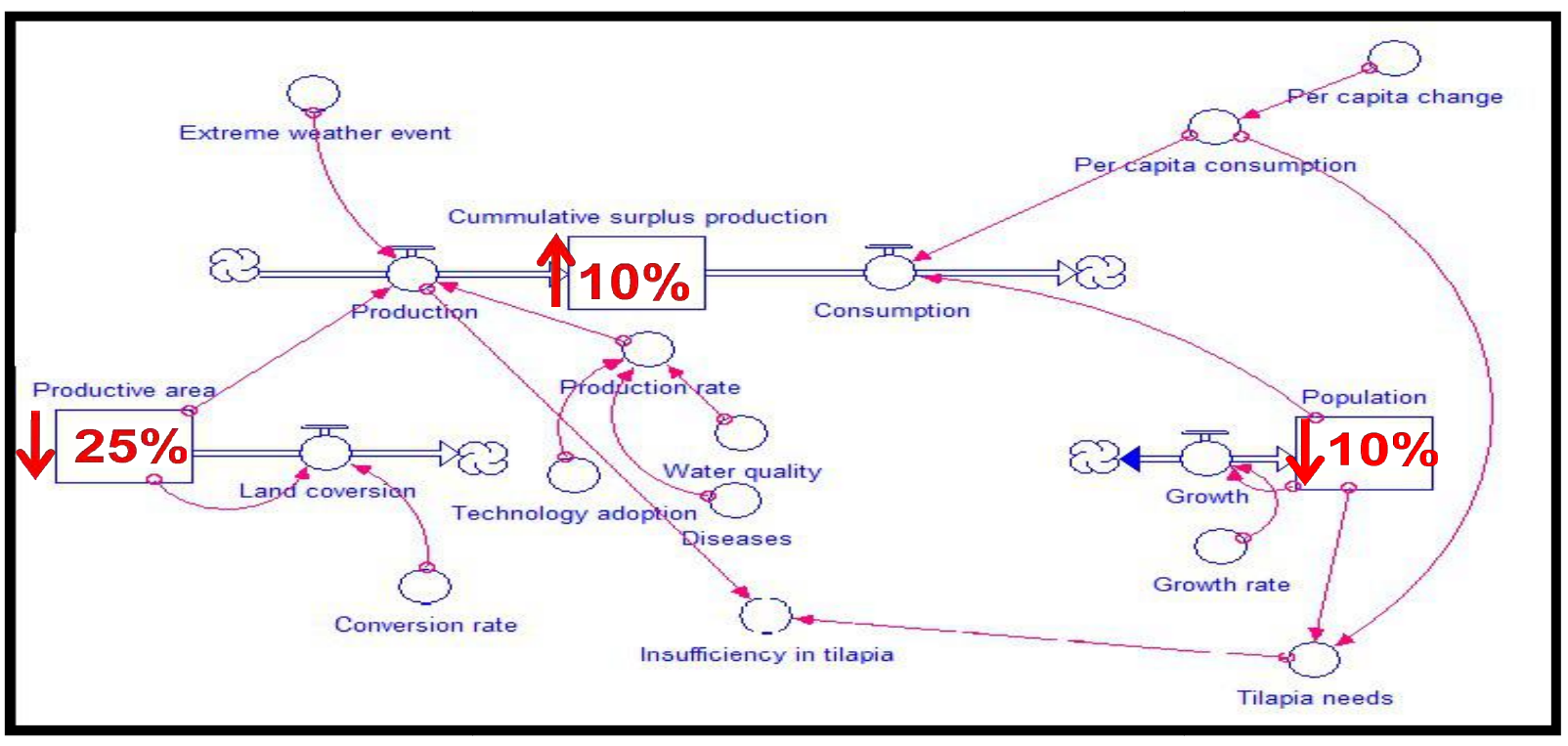

Figure 5: Local tilapia sufficiency model with intervention in productive area, production rate and population growth rate

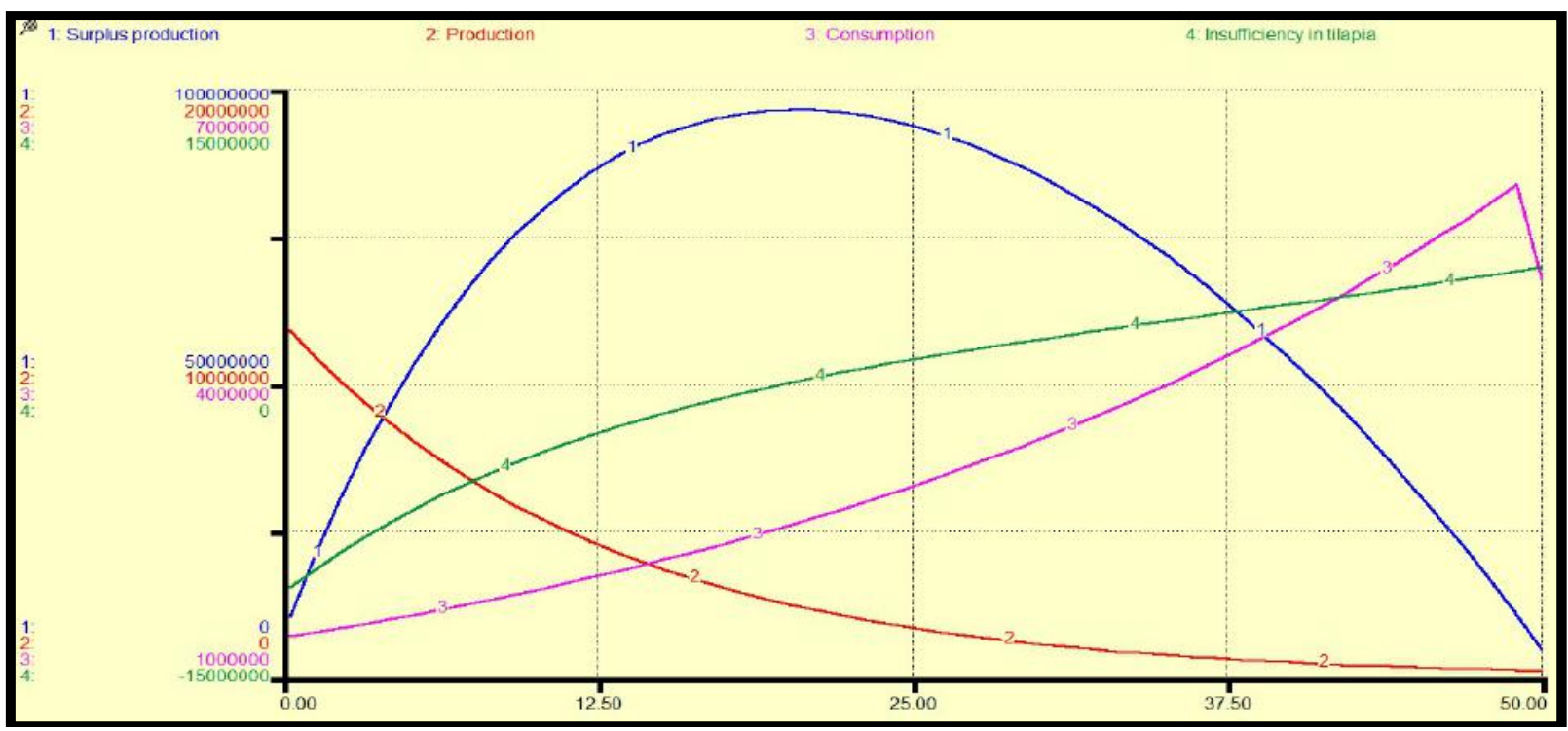

Figure 6: Improvement of local tilapia sufficiency due to added interventions 


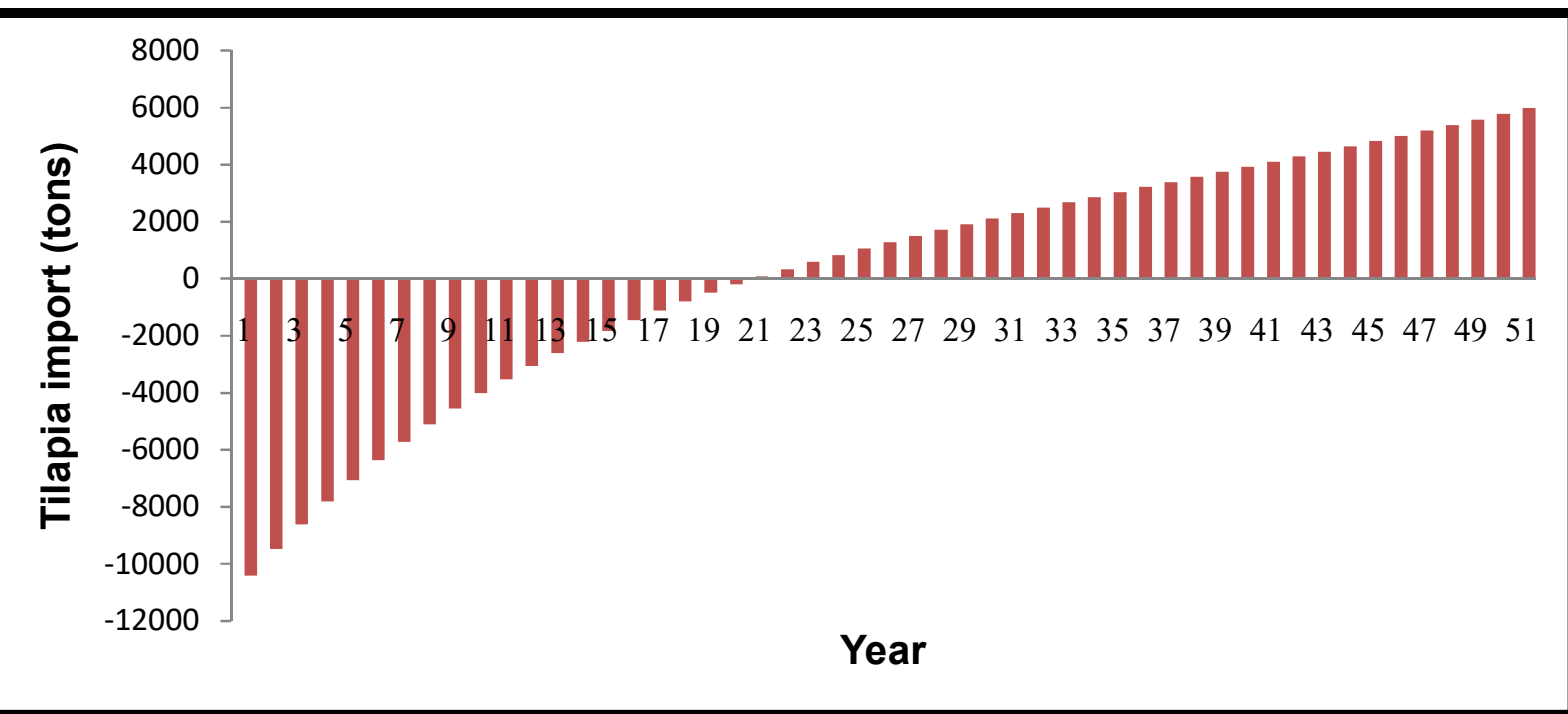

Figure 7: The municipality will start to import tilapia on the $20^{\text {th }}$ year

Table 3: Tabular result of the model with interventions $(10 \%$ technology adoption, $10 \%$ reduction population growth rate and $25 \%$ reduction land conversion rate)

\begin{tabular}{|c|c|c|c|c|c|}
\hline Year & $\begin{array}{l}\text { Productive } \\
\text { Area (ha) }\end{array}$ & $\begin{array}{l}\text { Production } \\
\text { (kg) }\end{array}$ & Consumption (kg) & $\begin{array}{l}\text { Cumulative Surplus } \\
\text { Production (kg) }\end{array}$ & Insufficiency (kg) \\
\hline 1 & $1,196.10$ & $10,936,041.86$ & $1,461,150.01$ & $20,808,311.43$ & $-9,474,891.85$ \\
\hline 3 & $1,023.41$ & $9,357,150.82$ & $1,550,134.04$ & $38,894,057.49$ & $-7,807,016.77$ \\
\hline 4 & 946.66 & $8,655,364.50$ & 1,596,638.06 & $46,701,074.27$ & $-7,058,726.44$ \\
\hline 5 & 875.66 & $8,006,212.17$ & $1,644,537.21$ & $53,759,800.71$ & $-6,361,674.96$ \\
\hline 8 & 693.04 & $6,336,541.64$ & $1,797,030.21$ & $70,938,974.36$ & $-4,539,511.43$ \\
\hline 9 & 641.06 & $5,861,301.02$ & $1,850,941.11$ & $75,478,485.79$ & $-4,010,359.90$ \\
\hline 10 & 592.98 & $5,421,703.44$ & $1,906,469.35$ & $79,488,845.69$ & $-3,515,234.09$ \\
\hline 11 & 548.51 & $5,015,075.68$ & $1,963,663.43$ & $83,004,079.79$ & $-3,051,412.25$ \\
\hline 12 & $\mathbf{5 0 7 . 3 7}$ & $4,638,945.01$ & $2,022,573.33$ & $86,055,492.04$ & $-2,616,371.68$ \\
\hline 17 & 343.59 & $3,141,433.62$ & $2,344,716.83$ & $95,284,193.98$ & $-796,716.80$ \\
\hline 18 & 317.82 & $2,905,826.10$ & $2,415,058.33$ & $96,080,910.78$ & $-490,767.77$ \\
\hline 19 & 293.98 & $2,687,889.14$ & $2,487,510.08$ & $96,571,678.55$ & $-200,379.06$ \\
\hline 20 & 271.93 & $2,486,297.46$ & $2,562,135.38$ & $96,772,057.61$ & $75,837.92$ \\
\hline 21 & 251.54 & $2,299,825.15$ & $2,638,999.44$ & $96,696,219.69$ & $339,174.30$ \\
\hline 22 & 232.67 & $2,127,338.26$ & $2,718,169.43$ & $96,357,045.39$ & $590,831.16$ \\
\hline 23 & 215.22 & $1,967,787.89$ & $2,799,714.51$ & $95,766,214.23$ & $831,926.62$ \\
\hline 24 & 199.08 & $1,820,203.80$ & $2,883,705.94$ & $94,934,287.61$ & $1,063,502.14$ \\
\hline 25 & 184.15 & $1,683,688.52$ & $2,970,217.12$ & $93,870,785.47$ & $1,286,528.61$ \\
\hline 26 & 170.34 & $1,557,411.88$ & $3,059,323.64$ & $92,584,256.86$ & $1,501,911.76$ \\
\hline 27 & 157.56 & $1,440,605.99$ & $3,151,103.35$ & $91,082,345.10$ & $1,710,497.36$ \\
\hline
\end{tabular}




\begin{tabular}{|c|c|c|c|c|c|}
\hline 36 & $\mathbf{7 8 . 1 1}$ & $\mathbf{7 1 4 , 2 0 1 . 5 9}$ & $\mathbf{4 , 1 1 1 , 4 7 5 . 1 5}$ & $\mathbf{6 8 , 7 5 5 , 3 4 3 . 8 0}$ & $\mathbf{3 , 3 9 7 , 2 7 3 . 5 6}$ \\
\hline 37 & $\mathbf{7 2 . 2 6}$ & $\mathbf{6 6 0 , 6 3 6 . 4 7}$ & $\mathbf{4 , 2 3 4 , 8 1 9 . 4 0}$ & $\mathbf{6 5 , 3 5 8 , 0 7 0 . 2 4}$ & $\mathbf{3 , 5 7 4 , 1 8 2 . 9 3}$ \\
\hline 38 & $\mathbf{6 6 . 8 4}$ & $\mathbf{6 1 1 , 0 8 8 . 7 3}$ & $\mathbf{4 , 3 6 1 , 8 6 3 . 9 8}$ & $\mathbf{6 1 , 7 8 3 , 8 8 7 . 3 1}$ & $\mathbf{3 , 7 5 0 , 7 7 5 . 2 5}$ \\
\hline 39 & $\mathbf{6 1 . 8 2}$ & $\mathbf{5 6 5 , 2 5 7 . 0 8}$ & $\mathbf{4 , 4 9 2 , 7 1 9 . 9 0}$ & $\mathbf{5 8 , 0 3 3 , 1 1 2 . 0 6}$ & $\mathbf{3 , 9 2 7 , 4 6 2 . 8 2}$ \\
\hline 40 & $\mathbf{5 7 . 1 9}$ & $\mathbf{5 2 2 , 8 6 2 . 8 0}$ & $\mathbf{4 , 6 2 7 , 5 0 1 . 5 0}$ & $\mathbf{5 4 , 1 0 5 , 6 4 9 . 2 3}$ & $\mathbf{4 , 1 0 4 , 6 3 8 . 7 0}$ \\
\hline 41 & $\mathbf{5 2 . 9}$ & $\mathbf{4 8 3 , 6 4 8 . 0 9}$ & $\mathbf{4 , 7 6 6 , 3 2 6 . 5 4}$ & $\mathbf{5 0 , 0 0 1 , 0 1 0 . 5 3}$ & $\mathbf{4 , 2 8 2 , 6 7 8 . 4 6}$ \\
\hline 42 & $\mathbf{4 8 . 9 3}$ & $\mathbf{4 4 7 , 3 7 4 . 4 8}$ & $\mathbf{4 , 9 0 9 , 3 1 6 . 3 4}$ & $\mathbf{4 5 , 7 1 8 , 3 3 2 . 0 7}$ & $\mathbf{4 , 4 6 1 , 9 4 1 . 8 6}$ \\
\hline 43 & $\mathbf{4 5 . 2 6}$ & $\mathbf{4 1 3 , 8 2 1 . 3 9}$ & $\mathbf{5 , 0 5 6 , 5 9 5 . 8 3}$ & $\mathbf{4 1 , 2 5 6 , 3 9 0 . 2 1}$ & $\mathbf{4 , 6 4 2 , 7 7 4 . 4 4}$ \\
\hline 44 & $\mathbf{4 1 . 8 7}$ & $\mathbf{3 8 2 , 7 8 4 . 7 9}$ & $\mathbf{5 , 2 0 8 , 2 9 3 . 7 0}$ & $\mathbf{3 6 , 6 1 3 , 6 1 5 . 7 7}$ & $\mathbf{4 , 8 2 5 , 5 0 8 . 9 2}$ \\
\hline 45 & $\mathbf{3 8 . 7 3}$ & $\mathbf{3 5 4 , 0 7 5 . 9 3}$ & $\mathbf{5 , 3 6 4 , 5 4 2 . 5 2}$ & $\mathbf{3 1 , 7 8 8 , 1 0 6 . 8 6}$ & $\mathbf{5 , 0 1 0 , 4 6 6 . 5 9}$ \\
\hline 46 & $\mathbf{3 5 . 8 2}$ & $\mathbf{3 2 7 , 5 2 0 . 2 4}$ & $\mathbf{5 , 5 2 5 , 4 7 8 . 7 9}$ & $\mathbf{2 6 , 7 7 7 , 6 4 0 . 2 7}$ & $\mathbf{5 , 1 9 7 , 9 5 8 . 5 6}$ \\
\hline 47 & $\mathbf{3 3 . 1 3}$ & $\mathbf{3 0 2 , 9 5 6 . 2 2}$ & $\mathbf{5 , 6 9 1 , 2 4 3 . 1 5}$ & $\mathbf{2 1 , 5 7 9 , 6 8 1 . 7 2}$ & $\mathbf{5 , 3 8 8 , 2 8 6 . 9 4}$ \\
\hline 48 & $\mathbf{3 0 . 6 5}$ & $\mathbf{2 8 0 , 2 3 4 . 5 0}$ & $\mathbf{5 , 8 6 1 , 9 8 0 . 4 5}$ & $\mathbf{1 6 , 1 9 1 , 3 9 4 . 7 8}$ & $\mathbf{5 , 5 8 1 , 7 4 5 . 9 5}$ \\
\hline 49 & $\mathbf{2 8 . 3 5}$ & $\mathbf{2 5 9 , 2 1 6 . 9 1}$ & $\mathbf{6 , 0 3 7 , 8 3 9 . 8 6}$ & $\mathbf{1 0 , 6 0 9 , 6 4 8 . 8 3}$ & $\mathbf{5 , 7 7 8 , 6 2 2 . 9 5}$ \\
\hline Final & $\mathbf{2 6 . 2 2}$ & & & $\mathbf{4 , 8 3 1 , 0 2 5 . 8 8}$ & $\mathbf{5 , 9 7 9 , 1 9 9 . 4 1}$ \\
\hline
\end{tabular}

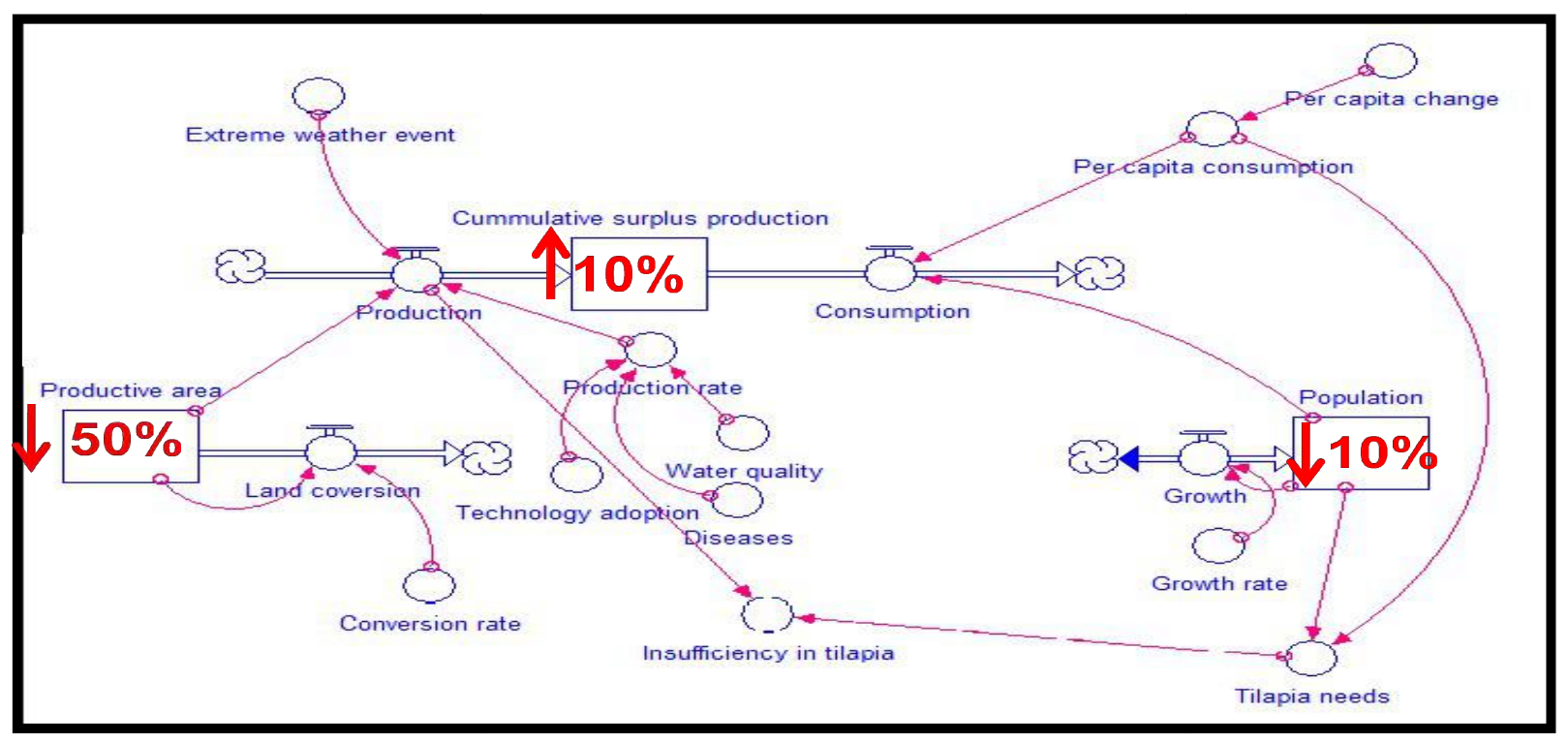

Figure 8: Local tilapia sufficiency model with $50 \%$ reduction in land conversion rate while other interventions remained constant 


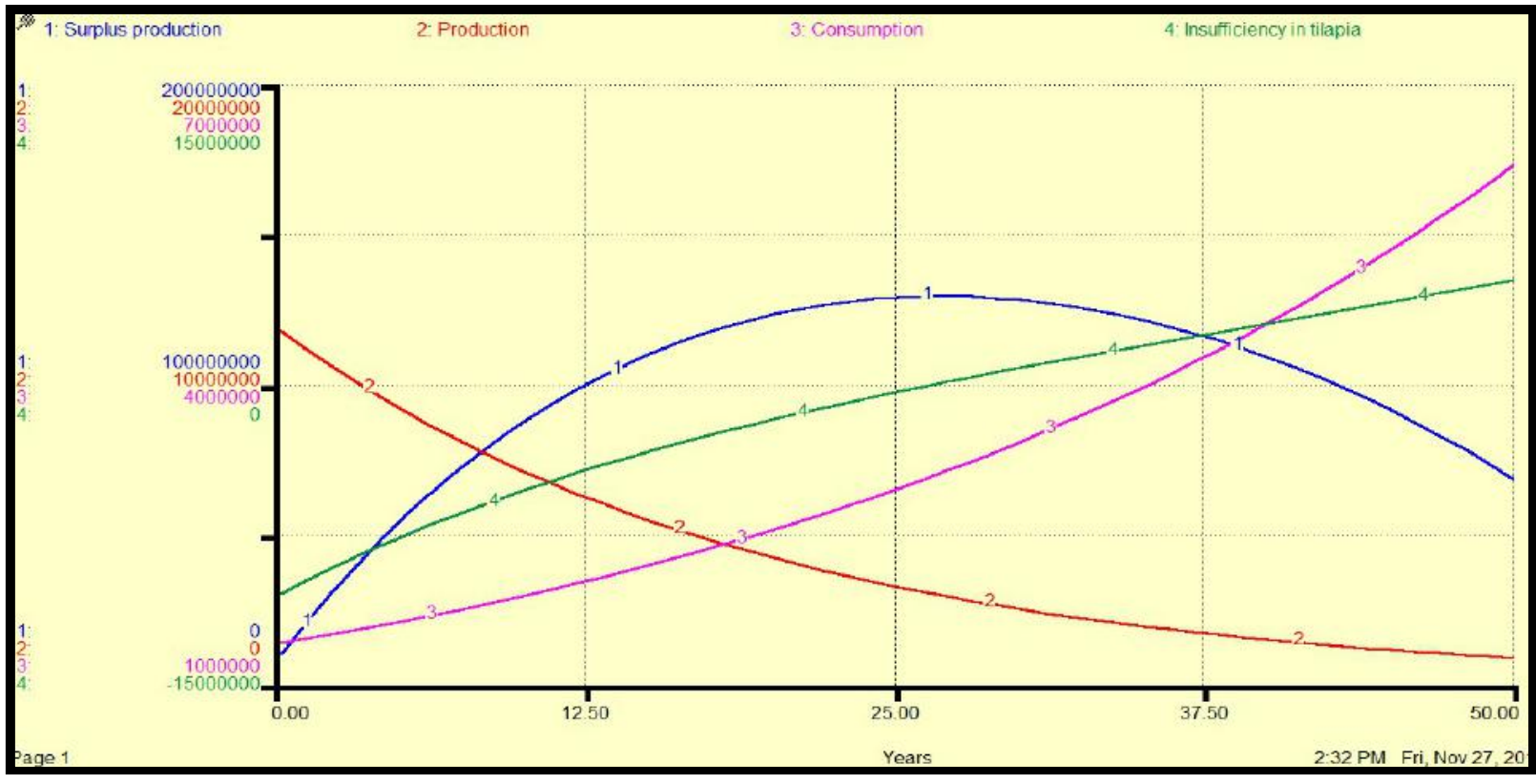

Figure 9: Reduction of land conversion rate up to $50 \%$ has resulted to a more sufficient supply of tilapia in the locality

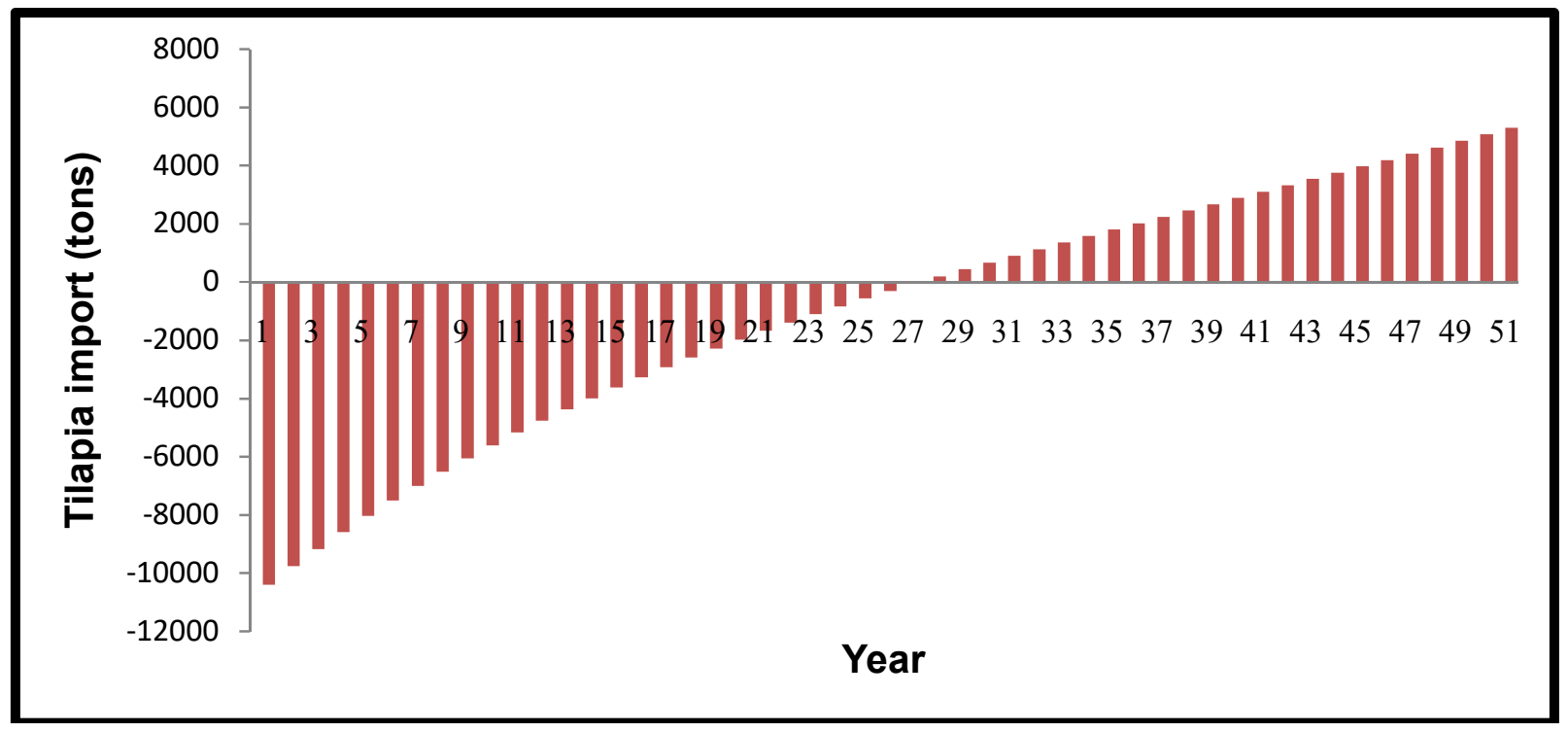

Figure 10: The municipality will start to import tilapia on the $27^{\text {th }}$ year 
Table 4: Tabular result of the model with interventions $(10 \%$ technology adoption, $10 \%$ reduction population growth rate and $50 \%$ reduction land conversion rate)

\begin{tabular}{|c|c|c|c|c|c|}
\hline Year & $\begin{array}{c}\text { Productive Area } \\
\text { (ha) }\end{array}$ & $\begin{array}{c}\begin{array}{c}\text { Production } \\
(\mathrm{kg})\end{array} \\
\end{array}$ & Consumption (kg) & $\begin{array}{c}\text { Cumulative Surplus } \\
\text { Production (kg) }\end{array}$ & Insufficiency (kg) \\
\hline $\mathbf{0}$ & $1,293.08$ & $11,822,747.96$ & $1,418,592.24$ & $10,404,155.72$ & $-10,404,155.72$ \\
\hline 1 & $1,228.43$ & $11,231,610.56$ & $1,461,150.01$ & $20,808,311.43$ & $-9,770,460.55$ \\
\hline 2 & $1,167.00$ & $10,670,030.03$ & $1,504,984.51$ & $30,578,771.98$ & $-9,165,045.52$ \\
\hline 3 & $1,108.65$ & $10,136,528.53$ & $1,550,134.04$ & $39,743,817.50$ & $-8,586,394.49$ \\
\hline 4 & $1,053.22$ & $9,629,702.10$ & $1,596,638.06$ & $48,330,211.99$ & $-8,033,064.04$ \\
\hline 5 & $1,000.56$ & $9,148,217.00$ & $1,644,537.21$ & $56,363,276.03$ & $-7,503,679.79$ \\
\hline 6 & 950.53 & $8,690,806.15$ & $1,693,873.32$ & $63,866,955.82$ & $-6,996,932.82$ \\
\hline 7 & 903.01 & $8,256,265.84$ & $1,744,689.52$ & $70,863,888.64$ & $-6,511,576.32$ \\
\hline 8 & 857.86 & $7,843,452.55$ & $1,797,030.21$ & $77,375,464.96$ & $-6,046,422.34$ \\
\hline 9 & 814.96 & $7,451,279.92$ & $1,850,941.11$ & $83,421,887.30$ & $-5,600,338.81$ \\
\hline 10 & 774.21 & $7,078,715.92$ & $1,906,469.35$ & $89,022,226.11$ & $-5,172,246.58$ \\
\hline 11 & 735.5 & $6,724,780.13$ & $1,963,663.43$ & $94,194,472.68$ & $-4,761,116.70$ \\
\hline 12 & 698.73 & $6,388,541.12$ & $2,022,573.33$ & $98,955,589.38$ & $-4,365,967.79$ \\
\hline 13 & 663.79 & $6,069,114.07$ & $2,083,250.53$ & 103,321,557.17 & $-3,985,863.54$ \\
\hline 14 & 630.6 & $5,765,658.36$ & $2,145,748.05$ & $107,307,420.71$ & $-3,619,910.32$ \\
\hline 15 & 599.07 & $5,477,375.44$ & $2,210,120.49$ & $110,927,331.03$ & $-3,267,254.96$ \\
\hline 16 & 569.12 & $5,203,506.67$ & $2,276,424.10$ & $114,194,585.98$ & $-2,927,082.57$ \\
\hline 17 & 540.66 & $4,943,331.34$ & $2,344,716.83$ & $117,121,668.55$ & $-2,598,614.51$ \\
\hline 18 & 513.63 & $4,696,164.77$ & $2,415,058.33$ & $119,720,283.06$ & $-2,281,106.44$ \\
\hline 19 & 487.95 & $4,461,356.53$ & $2,487,510.08$ & $122,001,389.51$ & $-1,973,846.45$ \\
\hline 20 & 463.55 & $4,238,288.71$ & $2,562,135.38$ & $123,975,235.96$ & $-1,676,153.32$ \\
\hline 21 & 440.37 & $4,026,374.27$ & $2,638,999.44$ & $125,651,389.28$ & $-1,387,374.83$ \\
\hline 22 & 418.35 & $3,825,055.56$ & $2,718,169.43$ & $127,038,764.11$ & $-1,106,886.13$ \\
\hline 23 & 397.44 & $3,633,802.78$ & $2,799,714.51$ & $128,145,650.24$ & $-834,088.27$ \\
\hline 24 & 377.57 & $3,452,112.64$ & $2,883,705.94$ & $128,979,738.51$ & $-568,406.70$ \\
\hline 25 & 358.69 & $3,279,507.01$ & $2,970,217.12$ & $129,548,145.21$ & $-309,289.89$ \\
\hline 26 & 340.75 & $3,115,531.66$ & $3,059,323.64$ & $129,857,435.09$ & $-56,208.02$ \\
\hline 27 & 323.71 & $2,959,755.08$ & $3,151,103.35$ & $129,913,643.11$ & $191,348.27$ \\
\hline 28 & 307.53 & $2,811,767.32$ & $3,245,636.45$ & $129,722,294.84$ & $433,869.13$ \\
\hline 29 & 292.15 & $2,671,178.96$ & $3,343,005.54$ & $129,288,425.72$ & $671,826.58$ \\
\hline 30 & 277.55 & $2,537,620.01$ & $3,443,295.71$ & $128,616,599.13$ & $905,675.70$ \\
\hline 31 & 263.67 & $2,410,739.01$ & $3,546,594.58$ & $127,710,923.43$ & $1,135,855.57$ \\
\hline 32 & 250.48 & $2,290,202.06$ & $3,652,992.41$ & $126,575,067.86$ & $1,362,790.36$ \\
\hline 33 & 237.96 & $2,175,691.95$ & $3,762,582.19$ & $125,212,277.51$ & $1,586,890.23$ \\
\hline 34 & 226.06 & $2,066,907.36$ & $3,875,459.65$ & $123,625,387.27$ & $1,808,552.30$ \\
\hline 35 & 214.76 & $1,963,561.99$ & $3,991,723.44$ & $121,816,834.98$ & $2,028,161.45$ \\
\hline 36 & 204.02 & $1,865,383.89$ & $4,111,475.15$ & $119,788,673.52$ & $2,246,091.26$ \\
\hline 37 & 193.82 & $1,772,114.69$ & $4,234,819.40$ & $117,542,582.27$ & $2,462,704.71$ \\
\hline 38 & 184.13 & $1,683,508.96$ & $4,361,863.98$ & $115,079,877.56$ & $2,678,355.02$ \\
\hline 39 & 174.92 & $1,599,333.51$ & $4,492,719.90$ & $112,401,522.54$ & $2,893,386.39$ \\
\hline 40 & 166.18 & $1,519,366.84$ & $4,627,501.50$ & $109,508,136.15$ & $3,108,134.66$ \\
\hline 41 & 157.87 & $1,443,398.49$ & $4,766,326.54$ & $106,400,001.49$ & $3,322,928.05$ \\
\hline 42 & 149.97 & $1,371,228.57$ & $4,909,316.34$ & $103,077,073.44$ & $\mathbf{3 , 5 3 8 , 0 8 7 . 7 7}$ \\
\hline 43 & 142.48 & $1,302,667.14$ & $5,056,595.83$ & $99,538,985.67$ & $3,753,928.69$ \\
\hline 44 & 135.35 & $1,237,533.78$ & $5,208,293.70$ & $95,785,056.98$ & $3,970,759.92$ \\
\hline 45 & 128.58 & $1, \mathbf{1 7 5 , 6 5 7 . 0 9}$ & $5,364,542.52$ & $91,814,297.06$ & $4,188,885.42$ \\
\hline 46 & 122.15 & $1,116,874.24$ & $5,525,478.79$ & $87,625,411.64$ & $4,408,604.55$ \\
\hline 47 & 116.05 & $1,061,030.53$ & $5,691,243.15$ & $83,216,807.09$ & $4,630,212.63$ \\
\hline 48 & 110.24 & $1,007,979.00$ & $5,861,980.45$ & $78,586,594.46$ & $4,854,001.45$ \\
\hline 49 & 104.73 & $957,580.05$ & $6,037,839.86$ & 73,732,593.01 & $5,080,259.81$ \\
\hline Final & 99.5 & & & $68,652,333.20$ & $5,309,274.01$ \\
\hline
\end{tabular}

The following are recommended for a long term tilapia sufficiency in Minalin, Pampanga: (1) As much as possible, land use conversion rate should be less than $5 \%$.
Evaluation of the latest CLUP of the municipality will give them a general view of the problem. In Pampanga, tilapia farms are usually converted to rice farm because of 
huge economic losses as caused by fish kill. The conversion could be indirectly lessened through providing technical and monetary assistance from the local and provincial government. These include provision of free fingerlings, holding of seminars to upgrade the skills and knowledge of the farmers, introduction of tested technologies in the farm level and many more; (2) The local and provincial government with the help of BFAR-Region III should tightly monitor the conditions of farm operation in order to maximize production. It is also helpful to establish a target yield per year to ensure tilapia sufficiency; (3) Strengthening the use of polyculture system should be an option. Tilapia could be communally reared with other protein-source commodities such as milkfish, shrimp or crab; (4) Establishment of post-harvest facilities is necessary for effective local utilization of the surplus produced tilapia; (5) It is essential that the municipal or provincial government has an environmental management plan that considers the disastrous effects of natural hazards and the potential aggravating effects of climate change. As much as possible, they should have a guideline on the construction of aquaculture facilities that could surmount flooding, typhoon and other hazards; and (6) Increasing awareness on family planning and delimiting migration in the municipality are possible ways of managing population growth rate.

\section{CONCLUSION}

Using STELLA Version 9.02, the factors affecting the self-sufficiency of tilapia in Minalin, Pampanga, Philippines were dynamically visualized. Available tilapia for local consumption was negatively affected by increasing population, change in capita consumption towards fish protein, reduction of available land for tilapia farming, poor water quality, diseases and extreme weather events such as typhoon. In order to lessen the compounding effects of the above mentioned factors, interventions such as technology adoption, management of population growth and reduction of land conversion rate were deemed effective. The local tilapia sufficiency was prolonged from 16 years to 19 years because of these interventions. In addition, 50\% reduction in land conversion rate could extend the local sufficiency of tilapia until the $26^{\text {th }}$ year.

\section{ACKNOWLEDGEMENT}

The author expressed his profound gratitude to the Department of Science and Technology (DOST), Dr. Emmanuel Vera Cruz, Mr. Roberto Miguel Sayco, Mr. Joel 
Sumeldan, Mr. Allan Botch Valdez, Mr. Mar Macasiray, Mr. Rodolfo Atayde, Ms. Annieraj Antong, Ms. Maria Theresa Llamas, Mr. Mark Joseph Mercado, Mr. Allan Mesina and Mr. John Benrich Zuñiga for making this study possible.

\section{REFERENCES}

[1] Food and Agriculture Organization of the United Nations. 2013.State of world aquaculture 2013. Retrieved from

http://www.fao.org/docrep/011/i0250 e/i0250e00.HTM on March 1, 2013.

[2] Naylor, R. and M. Burke. 2005. Aquaculture and ocean resources: raising tigers of the sea. Annual Review of Environmental Resources, 30:185-218.

Retrieved fromhttp://www.annualreviews.org/d oi/pdf/10.1146/annurev.energy.30.08 1804.121034on July 112012.

[3] Bureau of Agricultural Statistics, Department of Agriculture. 2006. Fisheries statistics of the Philippines. Volume 15.

[4] World Health Organization. 2003. The world health report, a safer future. Global public health security in the $21^{\text {st }}$ century. Geneva, Switzerland.

[5] Sarker, P.K., Gamble M.M., Kelson S. and Kapuscinski A.R. 2016. Nile tilapia (Oreochromis niloticus) show high digestibility of lipid and fatty acids from marine Schizochytrium sp. and of protein and essential amino acids from freshwater Spirulina sp. feed ingredients. Aquaculture Nutrition, 22:109-119.

[6] Panorama Acuicola Magazine. 2003. Pampanga hailed as "tilapia" capital of the Philippines.http://www.panoramaacui cola.com/noticias/2005/10/28/pampan ga_hailed_as_tilapia_capital_of_the philippines.html.

[7] Vera Cruz, E.M. and Reyes, A.T. 2014. Development of comprehensive geo-referenced database for ecological risk analyses of tilapia pond culture in Pampanga. Central Luzon State University, Science City of Muñoz, Nueva Ecija. 